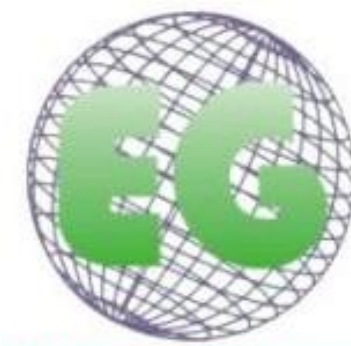

ISSN 1695-6141 $N^{\circ} 50$

\title{
La literatura como instrumento terapéutico en el proceso salud- enfermedad durante la infancia
}

Literature as a therapeutic instrument in the health-disease process in childhood

Izaro Babarro Vélez ${ }^{1}$
Jaione Lacalle Prieto

1 Doctoranda del programa de Psicología en el área de Psicobiología, Universidad del País Vasco/Euskal Herriko Unibertsitatea (UPV/EHU). España.

2 Profesora Adjunta de la Facultad de Medicina y Enfermería, Universidad del País Vasco/Euskal Herriko Unibertsitatea (UPV/EHU).España.

E-mail: jaione.lacalle@ehu.eus

\section{http://dx.doi.org/10.6018/eglobal.17.2.299201}

Recibido: 10/07/2017

Aceptado: 07/10/2017

\section{RESUMEN:}

Introducción: Todas las personas, a lo largo de la vida deben adecuarse a innumerables situaciones en las que se ven involucradas; es por ello que resulta necesario comprender cada una de ellas para poder avanzar adecuadamente. Se ha observado que las terapias psicológicas, entre las que se encuentra la biblioterapia, son efectivas para comprender los cambios a los que se enfrentan las personas.

Objetivo: Analizar y destacar los efectos más relevantes de la utilización de la literatura en el proceso salud-enfermedad del niño en diferentes momentos y contextos.

Método: Se ha realizado una revisión de la literatura mediante la búsqueda bibliográfica en las bases de datos Dialnet, Cuiden Plus, LILACS, Medline, Proquest, Pubmed, Scielo, Scopus, TDR, Trip y Biblioteca Virtual de la Salud.

Resultados: Tras la búsqueda bibliográfica se identificaron 2018 artículos de los cuales, tras la aplicación de filtros y criterios de inclusión, se obtuvieron 71 artículos. Finalmente se seleccionaron 26 artículos.

Conclusiones: La biblioterapia resulta beneficiosa en diversos ámbitos relacionados con el proceso salud-enfermedad durante la infancia, mejorando entre otras cosas la autoestima y la aceptación de la realidad, promoviendo el bienestar psicológico y sirviendo de puente en la comunicación entre el individuo y el profesional de la salud.

Palabras clave salud; niño; cuento; biblioterapia; enfermería y cuidados

\section{ABSTRACT:}

Introduction: Everybody, throughout their life, must adapt to countless situations in which they are involved; that is why it is necessary to understand each of them in order to advance properly. It has been observed that psychological therapies, among which bibliotherapy is, are effective to understand the changes that people face. 
Objective: To analyze and highlight the most relevant effects of the use of literature on the child's health-disease process at different times and contexts.

Method: A review of the literature was performed through the bibliographic research in the databases Dialnet, Cuiden Plus, LILACS, Medline, Proquest, Pubmed, Scielo, Scopus, TDR, Trip and Virtual Health Library.

Results: After the bibliographic research, 2018 articles were identified, out of which 71 articles were obtained after applying filters and inclusion criteria. Finally, 26 articles were selected.

Conclusions: Bibliotherapy is beneficial in various areas related to the health-disease process during childhood, improving among other things self-esteem and acceptance of reality, promoting psychological well-being and serving as a bridge in communication between the individual and the health professional.

Keywords: health; child; story; bibliotherapy; nursing care

\section{INTRODUCCIÓN}

La vida es una calle de sentido único y a medida que las personas realizan su recorrido, se encontrarán con diversos obstáculos a los que deberán enfrentarse. Los primeros pasos de este camino, que constituyen años, están llenos de cambios; esto conlleva que esta etapa de la infancia sea considerada en numerosas ocasiones novedosa a la par que preocupante ${ }^{(1)}$. Uno de los mejores recursos para resolver las dudas que puedan surgir a los más pequeños de la casa es el uso de la lectura.

La utilización de los libros, además de ser un objeto de entretenimiento, puede constituir una herramienta útil de ayuda en diversos procesos entre los que se encuentra el proceso de curación. El hecho de sanar por medio de los libros fue descrito por primera vez por S.M. Crothers en 1916 bajo el término "biblioterapia" en un artículo publicado por "The Atlantic Montly", aunque lo cierto es que la lectura ha estado presente en diferentes épocas y lugares a lo largo de la historia de la civilización ${ }^{(2)}$.

Así, en el antiguo Egipto, el faraón Ramses II agrupó ciertos libros de su biblioteca bajo el lema "Remedios para el alma" y en Roma, Aulo Cornelio Celso asoció la lectura al tratamiento médico. Del mismo modo los griegos se valieron de los libros como herramienta terapéutica médica y espiritual. A finales del siglo XVIII, Pinnel en Francia y Tuke en Inglaterra, comenzaron a ofrecer libros para sanar distintos tipos de dolencias. Los médicos del siglo XIX, por su parte, aseguraron que leer era uno de los mejores métodos para optimizar la situación de los pacientes internados ${ }^{(2,3)}$.

Otro avance significativo de la biblioterapia tuvo lugar a inicios del siglo XX, en el año 1930, cuando algunas bibliotecas comenzaron a ofrecer listados de libros adecuados a las características de cada persona ${ }^{(3)}$. En el año 2010 se creó una lista de libros bajo el término "Compassion Books", con la idea de ofrecer libros que tratasen de sanar, mediante el cuidado del alma, el dolor que sufría el cuerpo ${ }^{(2)}$.

A pesar de que se hayan asociado un sinfín de beneficios a la biblioterapia, esta técnica va más allá del simple hecho de la lectura de relatos. Por tanto, resulta necesario escoger adecuadamente las obras que se ajusten a cada situación personal y combinar dichos relatos con la reflexión, ya que sólo de esta manera se conseguirán los efectos deseados ${ }^{(1,4)}$.

De este modo, no se ofrecerá el mismo libro a una persona sana o que padezca una particular dolencia, ni tampoco a un adulto que a un niño. En el caso de los más jóvenes, los cuentos forman el eje de la biblioterapia ${ }^{(5-7)}$. 


\section{OBJETIVO}

Analizar los efectos de la biblioterapia en el proceso salud-enfermedad del niño.

\section{MÉTODO}

Se ha realizado una revisión narrativa con el fin de analizar los efectos de la literatura infantil a modo de recurso terapéutico.

La búsqueda bibliográfica se ha llevado a cabo en las siguientes bases de datos: Dialnet, Cuiden Plus, LILACS, Medline, Proquest, Pubmed, Scielo, Scopus, TDR, Trip y en Biblioteca Virtual de la Salud. En función de las características de cada una de las bases de datos, se han utilizado los siguientes Descriptores en Ciencias de la Salud (DeCS) y Medical Subject Headings (MeSH):

- DeCS: salud, niño, cuento, biblioterapia, enfermería y cuidados.

- MeSH: children, health, stories, bibliotherapy, nursing care.

Estas palabras clave han sido combinadas utilizando los operadores booleanos "AND", "NOT" y "OR".

Asimismo, los filtros utilizados han sido los siguientes: artículos publicados en los últimos 10 años y como idiomas, el castellano y el inglés.

Además se han definido varios criterios de inclusión y exclusión (véase tabla 1).

Tabla 1: Criterios de inclusión y exclusión.

\begin{tabular}{|l|l|}
\hline \multicolumn{1}{|c|}{ CRITERIOS DE INCLUSIÓN } & \multicolumn{1}{|c|}{ CRITERIOS DE EXCLUSIÓN } \\
\hline $\begin{array}{l}\text { Documentos que analizan la } \\
\text { utilización de la biblioterapia en la } \\
\text { infancia. }\end{array}$ & $\begin{array}{l}\text { Documentos que se centran en el } \\
\text { uso de la biblioterapia durante la } \\
\text { adolescencia o la edad adulta. }\end{array}$ \\
\hline $\begin{array}{l}\text { Trabajos que relacionan el uso de } \\
\text { la biblioterapia con el proceso } \\
\text { salud-enfermedad del niño y cómo } \\
\text { viven esto los distintos } \\
\text { profesionales. }\end{array}$ & $\begin{array}{l}\text { Trabajos que analizan el uso de } \\
\text { terapias que no están relacionadas } \\
\text { con la lectura. }\end{array}$ \\
\hline $\begin{array}{l}\text { Artículos que analizan la } \\
\text { importancia de la lectura a modo de } \\
\text { recurso educativo para la salud. }\end{array}$ & $\begin{array}{l}\text { Artículos que estudian otras } \\
\text { terapias cognitivo-conductuales a } \\
\text { modo de recurso educativo. }\end{array}$ \\
\hline $\begin{array}{l}\text { Trabajos que relacionan la } \\
\text { biblioterapia con la educación para } \\
\text { salud. }\end{array}$ & $\begin{array}{l}\text { Trabajos que analizan la } \\
\text { biblioterapia con el ámbito } \\
\text { pedagógico. }\end{array}$ \\
\hline
\end{tabular}

\section{RESULTADOS}

Las diferentes estrategias de búsqueda utilizadas en cada una de las bases de datos y los resultados de la búsqueda bibliográfica están reflejados en la Tabla 2. 
Tabla 2: Resultados de las búsquedas.

bibliográficas.

\begin{tabular}{|c|c|c|c|c|c|}
\hline $\begin{array}{l}\text { BASE DE } \\
\text { DATOS }\end{array}$ & $\begin{array}{l}\text { PALABRAS } \\
\text { CLAVE }\end{array}$ & $\begin{array}{l}\text { ART } \\
1 \text { (1) }\end{array}$ & FILTROS & $\begin{array}{l}\text { ART } \\
2 \text { (2) }\end{array}$ & $\begin{array}{c}\text { ARTICULOS } \\
\text { SELECCIONADOS }\end{array}$ \\
\hline BVS & $\begin{array}{l}\text { Biblioterapia } \\
\text { AND Niño }\end{array}$ & 95 & $\begin{array}{l}\text { Texto completo, } \\
\text { idiomas, } \\
\text { pertenecientes al } \\
\text { ámbito de salud. }\end{array}$ & 4 & 3 \\
\hline CUIDEN & $\begin{array}{l}\text { Libros AND } \\
\text { Niño AND } \\
\text { Salud }\end{array}$ & 7 & $\begin{array}{l}\text { Idiomas, } \\
\text { pertenecientes al } \\
\text { ámbito de salud. }\end{array}$ & 7 & 4 \\
\hline DIALNET & $\begin{array}{l}\text { Libros AND } \\
\text { Niño AND NOT } \\
\text { Adult }\end{array}$ & 1682 & $\begin{array}{l}\text { Texto completo, } \\
\text { idiomas, } \\
\text { pertenecientes al } \\
\text { ámbito de salud. }\end{array}$ & 254 & 4 \\
\hline LILACS & $\begin{array}{l}\text { Biblioterapia } \\
\text { AND Children } \\
\text { AND NOT } \\
\text { Adult }\end{array}$ & 4 & $\begin{array}{l}\text { Idiomas, } \\
\text { pertenecientes al } \\
\text { ámbito de salud. }\end{array}$ & 2 & 1 \\
\hline MEDLINE & $\begin{array}{l}\text { Biblioterapia } \\
\text { AND Children } \\
\text { AND NOT } \\
\text { Adult }\end{array}$ & 55 & $\begin{array}{l}\text { Texto completo, } \\
\text { idiomas, } \\
\text { pertenecientes al } \\
\text { ámbito de salud. }\end{array}$ & 15 & 3 \\
\hline PROQUEST & $\begin{array}{l}\text { Bibliotherapy } \\
\text { AND Health } \\
\text { AND Children }\end{array}$ & 164 & $\begin{array}{l}\text { Idiomas, texto } \\
\text { completo, ámbito } \\
\text { de salud o } \\
\text { psicosocial. }\end{array}$ & 22 & 4 \\
\hline PUBMED & $\begin{array}{l}\text { Bibliotherapy } \\
\text { AND Health } \\
\text { AND Children } \\
\text { AND NOT } \\
\text { Adult }\end{array}$ & 31 & $\begin{array}{l}\text { Idiomas, } \\
\text { pertenecientes al } \\
\text { ámbito de salud. }\end{array}$ & 5 & 2 \\
\hline SCIELO & $\begin{array}{l}\text { Biblioterapia } \\
\text { AND Niño }\end{array}$ & 9 & $\begin{array}{c}\text { Idiomas, } \\
\text { pertenecientes al } \\
\text { ámbito de salud. }\end{array}$ & 4 & 1 \\
\hline SCOPUS & $\begin{array}{l}\text { Bibliotherapy } \\
\text { AND Children } \\
\text { AND Nursing }\end{array}$ & 33 & $\begin{array}{l}\text { Idiomas, } \\
\text { pertenecientes al } \\
\text { ámbito de salud. }\end{array}$ & 30 & 2 \\
\hline TDR & Biblioterapia & 1 & & 1 & 1 \\
\hline $\begin{array}{c}\text { TRIP } \\
\text { DATABASE }\end{array}$ & Bibliotherapy & 101 & $\begin{array}{l}\text { Texto completo, } \\
\text { idiomas, } \\
\text { pertenecientes al } \\
\text { ámbito de salud. }\end{array}$ & 54 & 1 \\
\hline
\end{tabular}

ART $1_{(1)}$ : Artículos resultantes de la combinación de palabras clave y operadores booleanos. ART $2_{(2)}$ : Artículos obtenidos tras la utilización de los filtros.

Tras la búsqueda bibliográfica se identificaron 2018 artículos de los cuales 376 cumplían los criterios de selección. De ellos, tras la revisión de los resúmenes, se obtuvieron 71 por cumplir con los criterios de inclusión. Finalmente se seleccionaron 26 artículos por presentar niveles adecuados de evidencia (ver Tabla 2 y Figura 1). 
Figura 1: Diagrama de la selección de documentos

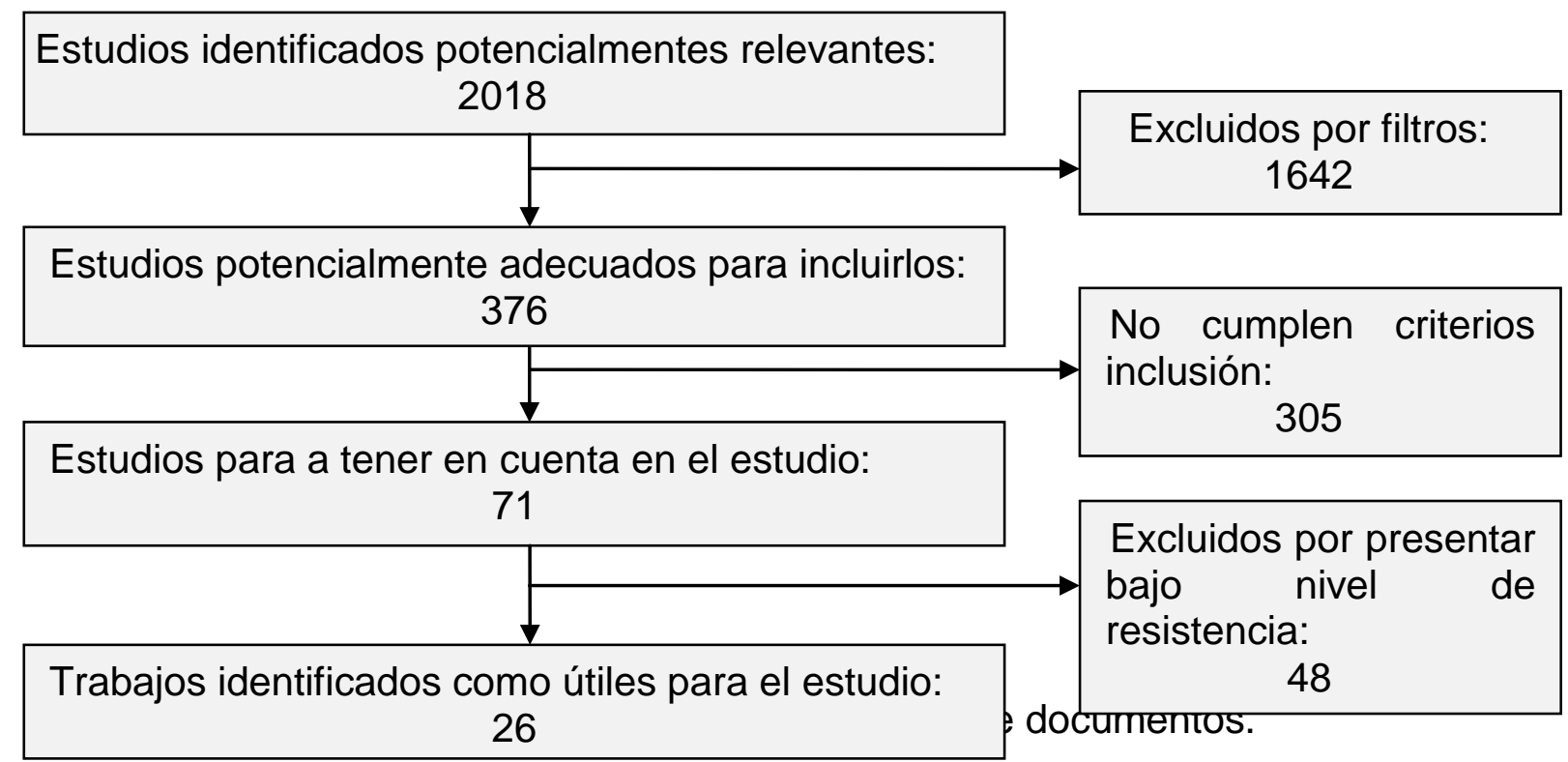

Tras realizar a cabo la selección de los artículos más pertinentes para la realización de esta revisión, se analizaron los 26 artículos obtenidos. En la Tabla 3 se muestran los resultados más relevantes de cada uno de estos artículos.

Tabla 3: Características más relevantes de los estudios.

\begin{tabular}{|c|c|c|c|}
\hline TÍTULO & $\begin{array}{l}\text { AUTORES } \\
\text { Y AÑO }\end{array}$ & $\begin{array}{l}\text { TIPO DE } \\
\text { ESTUDIO }\end{array}$ & $\begin{array}{l}\text { RESULTADOS MÁS } \\
\text { RELEVANTES }\end{array}$ \\
\hline $\begin{array}{l}\text { Afrontamiento } \\
\text { psicológico de los } \\
\text { procedimientos } \\
\text { médicos invasivos y } \\
\text { dolorosos aplicados } \\
\text { para el tratamiento del } \\
\text { cáncer infantil y } \\
\text { adolescente: la } \\
\text { perspectiva cognitivo- } \\
\text { conductual. }\end{array}$ & $\begin{array}{l}\text { Méndez et } \\
\text { al., } 2009\end{array}$ & $\begin{array}{l}\text { Revisión } \\
\text { narrativa }\end{array}$ & $\begin{array}{l}\text {-La lectura de } \text { cuentos } \\
\text { infantiles ayuda a los niños } \\
\text { pacientes oncológicos, a } \\
\text { enfrentarse a r sus } \\
\text { preocupaciones y miedos } \\
\text { relacionados con la } \\
\text { enfermedad, con los } \\
\text { procedimientos médicos y con } \\
\text { la hospitalización. } \\
\text {-La narración de historias } \\
\text { activa procesos de de } \\
\text { humanización, distracción y } \\
\text { aprendizaje. }\end{array}$ \\
\hline $\begin{array}{l}\text { A "novel" intervention: } \\
\text { a pilot study of } \\
\text { children's literature } \\
\text { and healthy lifestyles. }\end{array}$ & $\begin{array}{l}\text { Bravender } \\
\text { et al., } 2014\end{array}$ & $\begin{array}{l}\text { Ensayo } \\
\text { clínico }\end{array}$ & $\begin{array}{l}\text {-La lectura fomenta los } \\
\text { cambios en el estilo de vida, } \\
\text { favoreciendo la actividad } \\
\text { física y reduciendo el } \\
\text { consumo de alimentos ricos } \\
\text { en grasa y bebidas } \\
\text { azucaradas, incluso llegando } \\
\text { a la reducción del IMC (índice } \\
\text { de masa corporal). }\end{array}$ \\
\hline
\end{tabular}




\begin{tabular}{|c|c|c|c|}
\hline $\begin{array}{l}\text { Aprendiendo de mi } \\
\text { salud entre contextos } \\
\text { y cuentos. }\end{array}$ & $\begin{array}{l}\text { Moreno et } \\
\text { al., } 2009\end{array}$ & $\begin{array}{l}\text { Estudio } \\
\text { cualitativo }\end{array}$ & $\begin{array}{l}\text {-La lectura posibilita a los } \\
\text { niños a explorar y ampliar sus } \\
\text { conocimientos en torno al } \\
\text { medio hospitalario, a partir de } \\
\text { sus propias vivencias. }\end{array}$ \\
\hline $\begin{array}{l}\text { A prospective } \\
\text { randomised control } \\
\text { study: reduction of } \\
\text { children's pain } \\
\text { expectation using a } \\
\text { picture book during } \\
\text { blood withdrawal }\end{array}$ & $\begin{array}{l}\text { Zieger et al., } \\
2013\end{array}$ & $\begin{array}{l}\text { Ensayo } \\
\text { clínico }\end{array}$ & $\begin{array}{l}\text {-La lectura del cuento } \\
\text { favorece la reducción de las } \\
\text { expectativas de miedo ante } \\
\text { una extracción sanguínea. } \\
\text {-El uso de la anestesia local } \\
\text { interacciona con la eficacia } \\
\text { del cuento. }\end{array}$ \\
\hline $\begin{array}{l}\text { A randomized } \\
\text { controlled trial of } \\
\text { bibliotherapy for } \\
\text { carers of young } \\
\text { people with first- } \\
\text { episode psychosis }\end{array}$ & $\begin{array}{l}\text { McCann et } \\
\text { al., } 2013\end{array}$ & $\begin{array}{l}\text { Ensayo } \\
\text { clínico }\end{array}$ & $\begin{array}{l}\text {-La biblioterapia mejora la } \\
\text { experiencia de cuidado de } \\
\text { niños/as y adolescentes con } \\
\text { episodios de psicosis, } \\
\text { reduciendo los pensamientos } \\
\text { negativos y mejorando el } \\
\text { estrés. }\end{array}$ \\
\hline $\begin{array}{l}\text { Assessment and } \\
\text { management of } \\
\text { anxiety disorders in } \\
\text { children and } \\
\text { adolescents }\end{array}$ & $\begin{array}{l}\text { Creswell et } \\
\text { al., } 2014\end{array}$ & $\begin{array}{l}\text { Revisión } \\
\text { narrativa }\end{array}$ & $\begin{array}{l}\text {-Los problemas de ansiedad } \\
\text { son el trastorno psiquiátrico } \\
\text { más común durante la } \\
\text { infancia. } \\
\text {-Una de las maneras más } \\
\text { efectivas de tratar los } \\
\text { problemas de ansiedad es } \\
\text { mediante los libros, utilizando } \\
\text { la biblioterapia. }\end{array}$ \\
\hline $\begin{array}{l}\text { A story for children to } \\
\text { help children with HIV } \\
\text { understand the health- } \\
\text { disease process }\end{array}$ & $\begin{array}{l}\text { Brondani y } \\
\text { Pedro, } 2013\end{array}$ & $\begin{array}{l}\text { Investigación } \\
\text { cualitativa }\end{array}$ & $\begin{array}{l}\text {-El cuento "Peter's and Julia's } \\
\text { discovery: conversing about } \\
\text { health and disease" es un } \\
\text { recurso efectivo en niños con } \\
\text { VIH. Favorece la comprensión } \\
\text { de su situación y la } \\
\text { importancia del tratamiento. }\end{array}$ \\
\hline $\begin{array}{l}\text { Benefits of children's } \\
\text { and juvenile tales to } \\
\text { the hospitalized child } \\
\text { from the perspective } \\
\text { of nursing } \\
\text { professionals }\end{array}$ & $\begin{array}{l}\text { Rodrigues et } \\
\text { al., } 2012\end{array}$ & $\begin{array}{l}\text { Investigación } \\
\text { cualitativa }\end{array}$ & $\begin{array}{l}\text {-Los cuentos, al cumplir una } \\
\text { función lúdica, favorecen que } \\
\text { los niños olviden en parte la } \\
\text { condición de enfermedad. } \\
\text { - La lectura de cuentos mejora } \\
\text { la comunicación entre niño/a, } \\
\text { familia y profesionales de la } \\
\text { salud, del equipo de } \\
\text { enfermería en particular. }\end{array}$ \\
\hline $\begin{array}{l}\text { Bibliotherapy: a tool to } \\
\text { promote children's } \\
\text { psychological well- } \\
\text { being }\end{array}$ & $\begin{array}{l}\text { Vale y } \\
\text { Soares, } \\
2013\end{array}$ & $\begin{array}{l}\text { Revisión } \\
\text { narrativa }\end{array}$ & $\begin{array}{l}\text {-La literatura infantil, } \\
\text { promueve la mejora de } \\
\text { habilidades interpersonales, } \\
\text { la autoexpresión, el } \\
\text { autoconcepto e impulsa la } \\
\text { madurez emocional. }\end{array}$ \\
\hline
\end{tabular}




\begin{tabular}{|c|c|c|c|}
\hline $\begin{array}{l}\text { Brief report: a } \\
\text { "storybook" ending to } \\
\text { children's bedtime } \\
\text { problems-The use of a } \\
\text { rewarding social story } \\
\text { to reduce bedtime } \\
\text { resistance and } \\
\text { frequent night waking }\end{array}$ & $\begin{array}{l}\text { Burke et al., } \\
2004\end{array}$ & $\begin{array}{l}\text { Ensayo } \\
\text { clínico }\end{array}$ & $\begin{array}{l}\text {-La lectura de cuento "The } \\
\text { Sleep Fairy" reduce la } \\
\text { frecuencia de conductas y } \\
\text { despertares nocturnos, } \\
\text { manteniendo los resultados } \\
\text { durante tres meses. }\end{array}$ \\
\hline $\begin{array}{l}\text { Children's books for } \\
\text { use in Bibliotherapy. }\end{array}$ & $\begin{array}{l}\text { Tielsch, } \\
2011\end{array}$ & $\begin{array}{l}\text { Revisión } \\
\text { narrativa }\end{array}$ & $\begin{array}{l}\text {-La lectura fomenta el } \\
\text { desarrollo del niño y sirve de } \\
\text { puente para los profesionales, } \\
\text { promoviendo la comunicación. } \\
\text {-Ha resultado ser beneficiosa } \\
\text { en casos de "bullying", } \\
\text { ofreciendo herramientas a los } \\
\text { niños para lidiar con ello. } \\
\text {-"Compassion Books" lista de } \\
\text { libros de cuentos para ayudar } \\
\text { en la comprensión de } \\
\text { diversas situaciones. }\end{array}$ \\
\hline $\begin{array}{l}\text { Creating } \\
\text { bibliotherapeutic } \\
\text { libraries for pediatric } \\
\text { patients and their } \\
\text { families: potential } \\
\text { contributions of a } \\
\text { cognitive theory of } \\
\text { traumatic stress. }\end{array}$ & Waibel, 2010 & $\begin{array}{l}\text { Estudio } \\
\text { descriptivo }\end{array}$ & $\begin{array}{l}\text { - Las librerías terapéuticas son } \\
\text { efectivas para ofrecer soporte } \\
\text { psicológico a familias de niños } \\
\text { que han sufrido algún tipo de } \\
\text { episodio traumático. }\end{array}$ \\
\hline $\begin{array}{l}\text { "Érase una vez....un } \\
\text { cuento curativo". } \\
\text { Atención educativa en } \\
\text { población infantil } \\
\text { hospitalizada a través } \\
\text { de la literatura. }\end{array}$ & $\begin{array}{l}\text { Hérnandez y } \\
\text { Rabadán, } \\
2014\end{array}$ & $\begin{array}{l}\text { Revisión } \\
\text { narrativa }\end{array}$ & $\begin{array}{l}\text {-La lectura proporciona el } \\
\text { abordaje de una realidad } \\
\text { traumática, la continuación de } \\
\text { un ritmo de vida lo más } \\
\text { normalizado posible, la } \\
\text { superación de angustia ligada } \\
\text { a la hospitalización, ofrece } \\
\text { placer, ayuda en el desarrollo } \\
\text { personal y fomenta el } \\
\text { bienestar. }\end{array}$ \\
\hline $\begin{array}{l}\text { Evaluation of a } \\
\text { primary prevention } \\
\text { program for anxiety } \\
\text { disorders using story } \\
\text { books with children } \\
\text { aged } 9-12 \text { years. }\end{array}$ & $\begin{array}{l}\text { Bouchhard } \\
\text { et al., } 2013\end{array}$ & $\begin{array}{l}\text { Ensayo } \\
\text { clínico }\end{array}$ & $\begin{array}{l}\text {-La lectura de cuentos en } \\
\text { niños con problemas de } \\
\text { ansiedad, aporta nuevas } \\
\text { estrategias para aumentar las } \\
\text { habilidades de resolución de } \\
\text { problemas; mejorando los } \\
\text { niveles de ansiedad y la } \\
\text { sintomatología de estos niños. }\end{array}$ \\
\hline
\end{tabular}




\begin{tabular}{|c|c|c|c|}
\hline $\begin{array}{l}\text { Exploring the role of } \\
\text { digital storytelling in } \\
\text { pediatric oncology } \\
\text { patients' perspectives } \\
\text { regarding diagnosis: a } \\
\text { literature review }\end{array}$ & $\begin{array}{l}\text { Wilson et al., } \\
2015\end{array}$ & $\begin{array}{l}\text { Revisión } \\
\text { narrativa }\end{array}$ & $\begin{array}{l}\text {-Los libros resultan ser una } \\
\text { estrategia diferente para } \\
\text { entender la experiencia que } \\
\text { los niños están viviendo, } \\
\text { aportándoles la autonomía } \\
\text { necesaria para clarificar las } \\
\text { falsas creencias que suelen ir } \\
\text { asociadas a la enfermedad. } \\
\text {-En el caso de pacientes } \\
\text { oncológicos infantiles, los } \\
\text { cuentos favorecen la ruptura } \\
\text { de las barreras y ayudan a } \\
\text { que progresen en su proceso } \\
\text { y mejoren el comportamiento } \\
\text { en su entorno habitual. }\end{array}$ \\
\hline $\begin{array}{l}\text { Lectura de cuentos } \\
\text { infantiles como } \\
\text { estrategia de } \\
\text { humanización en el } \\
\text { cuidado del niño } \\
\text { encamado en } \\
\text { ambiente hospitalario }\end{array}$ & $\begin{array}{l}\text { Soares y } \\
\text { Correa, 2011 }\end{array}$ & $\begin{array}{l}\text { Investigación } \\
\text { cualitativa }\end{array}$ & $\begin{array}{l}\text {-La historia de "Buscando a } \\
\text { Nemo" favorece que los niños } \\
\text { con alguna minusvalía se } \\
\text { sientan identificados, } \\
\text { afrontando su realidad y } \\
\text { promoviéndoles a superar sus } \\
\text { límites. } \\
\text {-La lectura de cuentos } \\
\text { favorece la expresión de los } \\
\text { sentimientos, la mejora de las } \\
\text { reacciones del niño y sus } \\
\text { acompañantes, y proporciona } \\
\text { una actitud psicológica } \\
\text { positiva. }\end{array}$ \\
\hline $\begin{array}{l}\text { Leer para convivir. } \\
\text { Lecturas para la } \\
\text { prevención del acoso. }\end{array}$ & $\begin{array}{l}\text { Larrañaga et } \\
\text { al., } 2015\end{array}$ & $\begin{array}{l}\text { Ensayo } \\
\text { clínico }\end{array}$ & $\begin{array}{l}\text { - La lectura facilita la visión } \\
\text { integradora de la educación, } \\
\text { habiendo demostrado ser una } \\
\text { estrategia adecuada para } \\
\text { trabajar el acoso y potenciar } \\
\text { la empatía cognitiva. } \\
\text {-En españa un movimiento } \\
\text { "Cuentos para evitar el } \\
\text { bullying" y en Chile otro } \\
\text { movimiento denominado "iNo } \\
\text { al bullying!" con el fin de } \\
\text { realizar intervenciones en los } \\
\text { procesos de acoso escolar. }\end{array}$ \\
\hline $\begin{array}{l}\text { Los beneficios de la } \\
\text { lectura compartida de } \\
\text { libros: breve revisión. }\end{array}$ & $\begin{array}{l}\text { Goikoetxea y } \\
\text { Martinez, } \\
2015\end{array}$ & $\begin{array}{l}\text { Revisión } \\
\text { narrativa }\end{array}$ & $\begin{array}{l}\text {-La lectura compartida de } \\
\text { cuentos, además de favorecer } \\
\text { el desarrollo del lenguaje, } \\
\begin{array}{l}\text { potencia la la conexión } \\
\text { emocional. }\end{array}\end{array}$ \\
\hline
\end{tabular}




\begin{tabular}{|c|c|c|c|}
\hline $\begin{array}{l}\text { Percepción que el } \\
\text { niño/a tiene del "Diario } \\
\text { de Paula": Cuento } \\
\text { informativo sobre la } \\
\text { intervención } \\
\text { quirúrgica. }\end{array}$ & $\begin{array}{l}\text { Orihuela- } \\
\text { Pérez et al., } \\
2009\end{array}$ & $\begin{array}{l}\text { Estudio } \\
\text { descriptivo }\end{array}$ & $\begin{array}{l}\text { - La lectura del cuento "Diario } \\
\text { de Paula" favorece el } \\
\text { conocimiento del medio } \\
\text { hospitalario y la comprensión } \\
\text { del proceso quirúrgico como } \\
\text { una mejoría para la salud. } \\
\text {-El cuento como herramienta } \\
\text { lúdica y de educación para la } \\
\text { salud. }\end{array}$ \\
\hline $\begin{array}{l}\text { Portrayals of Bullying: } \\
\text { A content analysis of } \\
\text { picture books for } \\
\text { preschoolers. }\end{array}$ & $\begin{array}{l}\text { Oppliger y } \\
\text { Davis, } 2016\end{array}$ & $\begin{array}{l}\text { Revisión } \\
\text { narrativa }\end{array}$ & $\begin{array}{l}-91 \% \text { de las historias que } \\
\text { tratan de bullying aparecen } \\
\text { con la intención de parar el } \\
\text { acoso. } \\
-69 \% \text { de los cuentos que } \\
\text { tratan el acoso son narrados } \\
\text { desde el punto de vista de la } \\
\text { víctima. }\end{array}$ \\
\hline $\begin{array}{l}\text { La mediación de la } \\
\text { lectura como recurso } \\
\text { de comunicación con } \\
\text { niños hospitalizados }\end{array}$ & $\begin{array}{l}\text { Ceribelli et } \\
\text { al., } 2009\end{array}$ & $\begin{array}{l}\text { Estudio } \\
\text { cualitativo }\end{array}$ & $\begin{array}{l}\text {-La literatura infantil sirve de } \\
\text { herramienta en el proceso } \\
\text { salud-enfermedad del niño, } \\
\text { los efectos más destacables } \\
\text { son los siguientes: seguridad } \\
\text { emocional, disminución de } \\
\text { conflictos internos; } \\
\text { oportunidades para el } \\
\text { aprendizaje,la internalización } \\
\text { de valores humanos y } \\
\text { estrategias para superar } \\
\text { problemas. } \\
\text {-El cuento del "Patito Feo" } \\
\text { favorece la identificación con } \\
\text { el personaje en pacientes } \\
\text { oncohematológicos, } \\
\text { promoviendo el afrontamiento } \\
\text { de los cambios físicos.. }\end{array}$ \\
\hline $\begin{array}{l}\text { Storytelling as a } \\
\text { communication tool } \\
\text { for health consumers: } \\
\text { development of an } \\
\text { intervention for } \\
\text { parents of children } \\
\text { with croup. Stories to } \\
\text { communicate health } \\
\text { information }\end{array}$ & $\begin{array}{l}\text { Hartling et } \\
\text { al., } 2010\end{array}$ & $\begin{array}{l}\text { Ensayo } \\
\text { clínico }\end{array}$ & $\begin{array}{l}\text {-Los cuentos sirven de } \\
\text { herramienta para ofrecer } \\
\text { confort al niño e informar y } \\
\text { contextualizar la enfermedad. }\end{array}$ \\
\hline $\begin{array}{l}\text { Significados atribuidos } \\
\text { a la ocupación de la } \\
\text { lectura en el tiempo } \\
\text { libre. }\end{array}$ & $\begin{array}{l}\text { Rodríguez et } \\
\text { al., } 2011\end{array}$ & $\begin{array}{l}\text { Estudio } \\
\text { cualitativo }\end{array}$ & $\begin{array}{l}\text { - La lectura en niños/as ayuda } \\
\text { a disminuir el estrés y la } \\
\text { experiencia de dolor asociada } \\
\text { al medio hospitalario, así } \\
\text { como a mejorar la calidad de } \\
\text { vida. }\end{array}$ \\
\hline
\end{tabular}




\begin{tabular}{|c|c|c|c|}
\hline $\begin{array}{l}\text { The effectiveness of } \\
\text { creative bibliotherapy } \\
\text { for internalizing, } \\
\text { externalizing, and } \\
\text { prosocial behaviors in } \\
\text { children: a systematic } \\
\text { review. }\end{array}$ & $\begin{array}{l}\text { Montgomery } \\
\text { y Maunders, } \\
2015\end{array}$ & $\begin{array}{l}\text { Revisión } \\
\text { sistemática }\end{array}$ & $\begin{array}{l}\text {-La biblioterapia, favorece la } \\
\text { externalización de las } \\
\text { conductas; promueve la } \\
\text { conducta prosocial y la } \\
\text { empatía; reduce la ansiedad; } \\
\text { y ayuda a solventar } \\
\text { problemas sociales. }\end{array}$ \\
\hline $\begin{array}{l}\text { The impact of } \\
\text { bibliotherapy on } \\
\text { positive coping in } \\
\text { children who have } \\
\text { experienced disaster }\end{array}$ & $\begin{array}{l}\text { Pola y } \\
\text { Nelson, } \\
2014\end{array}$ & $\begin{array}{l}\text { Revisión } \\
\text { narrativa }\end{array}$ & $\begin{array}{l}\text { - La mayoría de estudios que } \\
\text { han analizado la efectividad } \\
\text { de la biblioterapia en } \\
\text { procesos traumáticos durante } \\
\text { la infancia han sido asociados } \\
\text { a desastres naturales, } \\
\text { ataques terroristas o guerras. }\end{array}$ \\
\hline $\begin{array}{l}\text { Treating nighttime } \\
\text { fears in young } \\
\text { children with } \\
\text { bibliotherapy: } \\
\text { evaluating anxiety } \\
\text { symptoms and } \\
\text { monitoring behavior } \\
\text { change }\end{array}$ & $\begin{array}{l}\text { Lewis et al., } \\
2015\end{array}$ & $\begin{array}{l}\text { Estudio } \\
\text { clínico }\end{array}$ & $\begin{array}{l}\text {-La lectura ha resultado ser } \\
\text { efectiva en la mejora de los } \\
\text { terrores nocturnos, así como } \\
\text { en los niveles de ansiedad, } \\
\text { las conductas evitativas y los } \\
\text { despertares nocturnos } \\
\text { asociados a dichos episodios. }\end{array}$ \\
\hline
\end{tabular}

Diversos estudios han demostrado la utilidad de la lectura en el desarrollo personal de los niños (ver tabla 3). Se diferencian distintos tipos de biblioterapia: la clínica (dirigida a enfermos y puesta en marcha por facultativos y bibliotecarios) y la evolutiva (bibliotecas escolares o públicas que promueven el autoconocimiento en diferentes etapas del desarrollo de la persona) ${ }^{(5)}$.

Tras analizar temáticamente las publicaciones seleccionadas, se han obtenido cinco categorías: "Niño sano", "Alteración en la esfera mental", "Hospitalización", "Patología en la esfera física" y "Fallecimiento".

\section{Niño sano}

Varios trabajos remarcan el papel de la lectura como una fuente de efectos beneficiosos para un niño que no presente ninguna patología pero que, a pesar de ello, debe ser capaz de adecuarse a diversas situaciones durante su desarrollo infantil. La lectura de relatos y su reflexión tienen efectos tales como reducir la ansiedad, favorecer la autoestima y la reflexión, ofrecer confort, ayudar a hacer frente a las adversidades y promover el desarrollo mediante la mejora de la comunicación y la externalización de los sentimientos ${ }^{(1-4)}$.

Se ha apreciado que la lectura estimula los estilos de vida saludables y puede prevenir diversas enfermedades tales como la obesidad y la diabetes infantil, llegando a conseguir que la lectura de cuentos favorezca la reducción del IMC (índice de masa corporal) $)^{(8)}$.

\section{Alteración en la esfera mental}

Las alteraciones mentales experimentadas en la infancia, implican que tanto el niño como su entorno tengan que ajustarse a la nueva realidad que están viviendo. 
Los terrores nocturnos y el miedo a la oscuridad, habituales en el desarrollo infantil, forman parte del grupo de las parasomnias, alteraciones que interfieren en la calidad del sueño. La biblioterapia promueve la aceptación de la fobia y una mayor adecuación e iniciativa al cambio por parte del niño y su familia, reduciendo así los niveles de ansiedad ${ }^{(9)}$. Otra problemática que con frecuencia aparece durante la infancia son las disrupciones de sueño y los despertares nocturnos. Se ha observado que esta problemática aparece en uno de cuatro niños/as antes de los 5 años. En ello, la lectura del cuento "The Sleep Fairy" ha resultado ser efectivo, mejorando las conductas asociadas a esta problemática ${ }^{(10)}$.

Por otro lado, el estrés postraumático es una situación que puede afectar a los niños en determinadas ocasiones. El uso de la literatura asociado a los procesos traumáticos se ha estudiado fundamentalmente en relación a desastres naturales, ataques terroristas o procesos de guerra ${ }^{(11)}$. Una situación traumática puede cambiar repentinamente la vida del niño, produciendo alteraciones en su desarrollo y afectando a la dinámica familiar. Mediante la creación de librerías infantiles, se ha procurado favorecer la asimilación de dicho episodio y sus secuelas. En un estudio realizado en Pensilvania (Waibel, 2010) se describe la creación de dos tipos de bibliotecas, una dirigida a casos en los que los niños han sido diagnosticados de alguna patología grave y otra adecuada a casos donde los niños han resultado ser víctimas sexuales o de violencia doméstica. Esta iniciativa promueve la adaptación a la nueva situación, mediante el compartir de las experiencias ${ }^{(12)}$.

La utilización de la lectura también se ha relacionado con el tratamiento de otro tipo de alteración que afecta a la salud mental, la psicosis. Este tipo de trastorno puede afectar, además de al niño que la padece, indirectamente a la salud de sus cuidadores. No existen muchos materiales para promover el cuidado de estos pacientes, pero se ha visto que el uso de la biblioterapia, a largo plazo, puede incluso ser más efectivo que el tratamiento médico habitual. Según McCann et al., los niños tratados con esta terapia valoran más positivamente los cambios vividos que los tratados con el tratamiento médico habitual ${ }^{(13)}$.

En procesos de ansiedad, alteración mental más frecuente en la infancia, la utilización de cuentos infantiles ha conseguido la mejoría de estos niños. La literatura infantil tiene como propósito la identificación del lector con el protagonista que lucha contra diferentes agentes estresores, consiguiendo así, que de la misma manera, ellos mismos sean capaces de enfrentarse al estrés que están viviendo de manera autónoma. La reflexión sobre estos cuentos también es necesaria para que los más pequeños aprendan a comportarse de manera adecuada ante futuras posibles situaciones $^{(14,15)}$.

El uso de la literatura infantil, además, puede resultar beneficioso para niños con capacidades especiales como es el caso de niños con Trastorno del Espectro Autista (TEA); trastorno psicológico y comportamental caracterizado por presentar alteraciones en la socialización con los demás. El cuento " $A$ friend like Simon: A story about autism" narra la historia de un niño con TEA desde el punto de vista de su mejor amigo y compañero de aula. El objetivo de este cuento es enseñar a los niños sobre las necesidades especiales que presentan las personas con TEA y la importancia de aceptar a todo el mundo por muy diferentes que éstos sean ${ }^{(2)}$.

Por último, una situación cada vez más común en los centros escolares y que se relaciona con el desarrollo de sintomatología relacionada con la salud mental es el bullying o acoso escolar. Varios estudios han demostrado la eficacia y la necesidad de 
tratar el acoso escolar mediante la biblioterapia. Existen diversos cuentos infantiles que se han utilizado con el fin de prevenir y dar otras perspectivas del acoso escolar $^{(16-17)}$.

\section{Hospitalización}

La enfermedad genera la ruptura del equilibrio que posee un niño hasta el momento de su aparición. En muchas ocasiones la pérdida de salud produce que el infante tenga que hacer frente a una situación estresante como es la hospitalización ${ }^{(17)}$.

El hospital constituye un entorno desconocido que conlleva cambios biopsicosociales y en el que una persona debe enfrentarse a técnicas dolorosas llevadas a cabo por personas ajenas a ella. Todo ello puede producir miedo, angustia, estrés e incluso sentimientos de abandono. Diversos autores proponen que los cuidados sanitarios deben ir más allá de las técnicas médicas; intentando humanizar estos cuidados, proponen la utilización de diversas terapias cognitivo-conductuales para cambiar la percepción que el infante y su familia tiene sobre la hospitalización. La biblioterapia se considera una herramienta que ofrece seguridad emocional, ayuda a superar el miedo a lo desconocido, fomenta el desarrollo personal y favorece el bienestar emocional, la reflexión y la construcción del criterio personal ${ }^{(5,10,11,18,19)}$. En cuanto a la relación profesional-usuario, la lectura de cuentos favorece el feedback y la comunicación. Además, ayuda a que el niño continúe con su proceso de escolarización, sirviendo no solo como método terapéutico, sino también, como recurso pedagógico ${ }^{(7,9,12,13,18-23)}$.

Una de las técnicas a la que con mayor frecuencia se exponen las personas en el ámbito sanitario es la extracción sanguínea. La lectura de un cuento ha resultado ser de utilidad en los niños en cuanto a la reducción y mejora de las expectativas de miedo asociadas a la extracción ${ }^{(4)}$.

Una persona puede requerir hospitalización por diversas razones, tanto por el hecho de padecer una patología crónica como aguda. Una de las razones más habituales entre las patologías agudas, en el caso de los más pequeños, es la amigdalitis o las anginas. Con el fin de ayudar en el proceso de esta patología, Hartling et al. evaluaron una intervención basada en la utilización de diversos cuentos tras lo cual señalaron los beneficios de esta herramienta ${ }^{(25)}$.

Otra razón de hospitalización en la infancia puede ser el hecho de enfrentarse a una intervención quirúrgica. Para hacer frente a esta situación Orihuela-Pérez et al. crearon y evaluaron la utilización de un cuento titulado "Diario de Paula", el cual ayuda tanto al niño como a su familia en la preparación de esta nueva experiencia ${ }^{(26)}$.

\section{Patología en la esfera física}

Una patología crónica es aquella enfermedad de larga duración y por lo general de progresión lenta. La lectura de cuentos a niños que sufren esta situación favorece la aceptación del diagnóstico, la adhesión al tratamiento y como consecuencia, una mayor calidad de vida ${ }^{(2)}$.

Una de las patologías pediátricas crónicas diagnosticada con más frecuencia es el cáncer, las leucemias en particular. Trabajar con un cuento como el "Patito Feo" ayuda al niño y a su familia a minimizar la afectación emocional y favorecer la confianza respecto al profesional de la salud. Este cuento narra la historia de un patito que es discriminado por sus iguales debido a su aspecto físico y con el paso del tiempo, se convierte en un hermoso cisne. Los niños tienden a identificarse con el 
protagonista, ya que, al igual que el patito feo, a consecuencia de la quimioterapia sufren un cambio físico notorio, derivado de la pérdida del cabello ${ }^{(14,23,24)}$.

Otra de las alteraciones crónicas presentes en la infancia son las diversas discapacidades. El relato "Buscando a Nemo" hace referencia a los procesos de minusvalía, ya que se basa en la historia de un pez que, aun teniendo una de sus aletas más corta, consigue con esfuerzo cumplir todos sus sueños y objetivos. Mediante esta historia, el niño puede interiorizar que, pese a tener una discapacidad, con esfuerzo, es capaz de hacer frente a los obstáculos que la propia vida impone, al igual que "Nemo" con su diminuta aleta ${ }^{(20)}$.

Una de las enfermedades infectocontagiosas más graves que puede aparecer durante la infancia es el VIH o la condición de SIDA en su evolución más crónica. Es una patología que resulta de difícil afrontamiento para el niño, entre otras cosas, por la estigmatización y estereotipos asociados que existen en la sociedad a cerca de las personas seropositivas, lo que puede afectar al entorno social del pequeño. En el año 2013 se realizó un estudio para evaluar la efectividad de un cuento para los niños que se encontraban en esta condición. Los resultados demostraron que el relato infantil "Peter's and Julia's Discovery: conversing about health and illness" es un recurso de utilidad que fomenta la conversación con los niños acerca del proceso saludenfermedad, favoreciendo la comprensión de su situación y la importancia del tratamiento antirretroviral ${ }^{(6)}$.

\section{Fallecimiento}

Diversos cuentos narran la realidad de los últimos días a los que tiene que hacer frente una persona. La lectura de cuentos como "Gentle Willow", donde una ardilla da su apoyo en los últimos días de vida al árbol que un día le había dado cobijo, constituye una herramienta de ayuda para que los niños lidien con sentimientos de miedo, enfado, dolor y frustración. De esta manera, se favorece que tanto ellos como sus familiares, se preparen mejor para la dura realidad del fin de una vida ${ }^{(8)}$.

\section{DISCUSIÓN}

Los resultados de la presente revisión revelan cuáles son los efectos asociados a la biblioterapia y su importancia. El $54,1 \%$ de los estudios se centran en estudiar los efectos de la biblioterapia en niños de edades entorno a los 2-16 años. Por otra parte 10 de los estudios, lo que supone un $41,6 \%$, se centran en analizar los efectos de la biblioterapia en niños en diferentes ámbitos sin especificar las edades. Y sólo uno se ha centrado en los efectos de la biblioterapia durante la adolescencia.

Respecto a la ciudad donde se realizó la investigación, la mitad de los estudios se realizaron en Estados unidos, 7 de ellos en países de Norteamérica, y 5 en Sudamérica. Sólo 4 se llevaron a cabo en Europa, de los cuales sólo 1 fue en España. En esta línea, cabe citar que se han escogido los estudios con el fin de mostrar la diversidad de la utilización de la biblioterapia en distintos ámbitos. En el $29,1 \%$ de los estudios seleccionados estudian la eficacia de la lectura de libros ligado al proceso de la hospitalización.

A pesar de haber demostrado la efectividad de la biblioterapia, sería aconsejable dar continuidad a esta terapia y que los profesionales que trabajen con niños en centros 
de salud, en hospitales o en distintas instituciones relacionadas con la salud, tuvieran conocimientos suficientes sobre los beneficios de los libros y pudieran así trabajar de una manera más adecuada con los más pequeños. Además, deberían de realizarse más estudios experimentales o cuasiexperimentales, ya que sólo el 37,5\%, 9 de los estudios, son ensayos clínicos, siendo la mayoría, el 54\%, revisiones o estudios descriptivos y cualitativos.

\section{CONCLUSIONES}

La utilización de la lectura como parte de la terapia cognitivo-conductual infantil es una herramienta que requiere precisión y conocimientos para su óptima implementación. Se considera necesario seguir unos pasos y una metodología adecuada, por lo que es imprescindible una adecuada formación para conseguir los efectos beneficiosos de la misma como son: reducir la ansiedad y el estrés, favorecer el diagnóstico de la enfermedad, ayudar la adherencia al tratamiento, mejorar la salud psicosocial y mejorar el bienestar y la calidad de vida. Además se sugiere la promoción de líneas de investigación relacionadas con el uso de la biblioterapia para poder obtener un mayor número de evidencias científicas que evalúen su calidad. La utilización de esta técnica podría favorecer la humanización de los cuidados, aspecto fundamental en el proceso salud-enfermedad, sobre todo cuando hablamos de los más pequeños, los cuales habitualmente carecen de los conocimientos y estrategias de afrontamiento adecuadas.

\section{REFERENCIAS}

(1) Montgomery P, Maunders K. The effectivenes of creative bibliotherapy for internalizing, externalizing, and prosocial behaviors in children: a systematic review. J Child Youth Serv Rev [Internet]. 2015 [acceso 25 de marzo de 2017]; 55: 37-47. Disponible en: http://dx.doi.org/10.1016/j.childyouth.2015.05.0100190-7409

(2) Tielsch A. Children's books for use in bibliotherapy. J Pediatr Health Car [Internet]. 2011 [fecha acceso 10 de marzo de 2017]; 25: 57-61. Disponible en: http://www.medscape.com/viewarticle/734236

(3) Goikoetxea E, Martínez N. Los beneficios de la lectura compartida de los libros: breve revisión. Educ XXI [Internet]. 2015 [acceso 10 de marzo de 2017]; 18(1):303-24. Disponible en: http://revistas.uned.es/index.php/educacionXX1/article/view/12334

(4) Zieger B, Praskova M, Busse E, Barth M. A prospective randomised control study: reduction of children's pain expectation using a picture book during blood withdrawal. Thieme [Internet]. 2013 [acceso 5 abril de 2017]; 1-5. Disponible en: http://dx.doi.org/10.1055/s-0033-1343481.

(5) Rodrigues EC, Dias EC, Menezes ES, Pinto P, Ferreira MA. Benefits of children's and juvenile tales to the hospitalized child from the perspective of nursing professionals. J Nurs UFPE [Internet]. 2012 [acceso 5 abril de 2017]; 6 (10): 2463-8. Disponible en: http://www.revista.ufpe.br/revistaenfermagem/index.php/revista/article/viewArticl e/3339

(6) Brondani JP, Pedro ENR. A story for children to help children with HIV understand the health-disease process. Rev Gaúch Enferm [Internet]. 2013 [acceso 10 de marzo de 2017]; 34(1):14-21. Disponible en: http://www.scielo.br/scielo.php?.script=sci serial\&pid=19831447\&lng=pt\&nrm=i $\underline{\text { so }}$ 
(7) Vale C, Soares L. Bibliotherappy: a tool to promote children's psychological wellbeing. J Poetry Therapy [Internet]. 2013 [acceso 10 de marzo de 2017]; 26(3): 137-47.

Disponible

en: http://www.tandfonline.com/doi/abs/10.1080/08893675.2013.823310?journalCo de=tjpt20

(8) Bravender T, Russell A, Chung RJ, Armstrong SC. A "Novel" Intervention: A pilot study of children's literature and healthy lifestyles. Pediatrics [Internet]. 2010 [acceso 15 de marzo de 2017];125(3): 513-7. Disponible en: http://pediatrics.aappublications.org/content/early/2010/02/08/peds.2009-1666

(9) Lewins KM, Amatya K, Coffman MF, Ollendick TH. Treating nightime fears in young children with bibliotherapy: Evaluating anxiety symptoms and monitoring behavior change. J Anxiety Disord [Internet]. 2015 [acceso 10 de marzo de 2017]; 30: 103-12. Disponible en: http://dx.doi.org/10.1016/.j.janxdis.2014.12.004

(10) Burke RV, Kuhn BR, Peterson JL. Brief report: a "storybook" ending to children's bedtime problems: the use of a rewarding social story to reduce bedtime resistance and frequent night walking. Journal Pediatr Psychol [Internet]. 2004 [acceso 4 de marzo de 2017]; 29(5):390-6. Disponible en: https://www.ncbi.nlm.nih.gov/pubmed/15187177

(11) Pola A, Nelson R. The Impact of Bibliotherapy on Positive Coping in Children Who Have Experienced Disaster. Therapeutic Recreation Journal [Internet]. 2014 [acceso 10 de marzo de 2017]; 48 (4): 341-44.

(12) Waibel MK. Creating Bibliotherapeutic Libraries for Pediatric Patients and Their Families: Potential Contributions of a Cognitive Theory of Traumatic Stress. J Pediatr Nurs [Internet]. 2010 [acceso 10 de marzo de 2017];25:25-7. Disponible en: http://www.pediatricnursing.org/article/S0882-5963(08)00311-4/abstract

(13) McCann TV, Lubman DI, Cotton SM, Murphy B, Crisp K, Catania L, Marck C, Gleeson JFM. A randomized controlled trial of bibliotherapy for carers of young people with first-episode psychosis. Schisophrenia Bull [Internet]. 2012 [acceso 24 de marzo de 2017]; 39(6): 1307-17. Dispsonible en: http://schizophreniabulletin.oxfordjournals.org/content/early/2012/11/20/schbul.s bs121

(14) Bouchard S, Gervais J, Loranger C. Evaluation of a primary prevention program for anxiety disorders using story books with children aged 9-12 years. J Primary Prev [Internet]. 2013 [acceso 10 de marzo de 2017]; 34: 345-58. Disponible en: https://www.ncbi.nlm.nih.gov/pubmed/23943134

(15) Creswell C, Waite P, Cooper PJ. Assesment and management of anxiety disorders in children and adolescents. J Arch Dis Child [Internet]. 2014 acceso 10 de marzo de 2017]; 99: 674-8. Disponible en: https://www.ncbi.nlm.nih.gov/pmc/articles/PMC4480225/pdf/nihms-700856.pdf

(16) Larrañaga $E$, Acosta M y Yubero S. Leer para convivir. Lecturas para la prevención del acoso. Educació Social [Internet]. 2015 [acceso 3 abril 2017]; 59: 71-85. Disponible en: http://www.observatoriodelainfancia.es/oia/esp/documentos ficha.aspx?id=521 7.

(17) Oppliger PA y Davis A. Portrayals of Bullying: A content analysis of pictures books for preschoolers. Early Childhood Educ J [Internet]. 2016 [acceso 7 de abril de 2017]; 44:515-26. Disponible en: https://link.springer.com/article/10.1007/s10643-015-0734-1

(18) Hernández E, Rabadán JA. "Erase una vez...un cuento curativo". Atención educativa en población infantil hospitalizada a través de la literatura. Educ.SXXI 
[Internet]. 2014 [acceso 24 de marzo de 2017]; 32(2): 129-50. Disponible en: http://revistas.um.es/educatio/article/view/194121

(19) Moreno E, Piedrahita AM, Hernández C. Aprendiendo de mi salud entre contextos y cuentos. Rev Educ y Pedagog [Internet]. 2009 [acceso 20 de marzo de 2017]; 21(53): 157-64. Disponible en: http://aprendeenlinea.udea.edu.co/revistas/index.php/revistaeyp/article/view/98 $\underline{52 / 9051}$

(20) Ortigosa JM, Méndez FX, Riquelme A. Afrontamiento psicológico de los procedimientos médicos invasivos y dolorosos aplicados para el tratamiento del cáncer infantil y adolescente: la perspectiva cognitivo-conductual. Psichooncologie [Internet]. 2009 [acceso 7 de abril de 2017]; 2(6): 413-28. Disponible en: http://revistas.ucm.es/index.php/PSIC/article/view/PSIC0909220413A

(21) Rodríguez MC, Teramelli IR, Torres V. Significados atribuidos a la ocupación de la lectura en el tiempo libre. Rev Astur [Internet].2011 [acceso 7 de marzo de 2017]; 9:17-23. Disponible en: https://dialnet.unirioja.es/servlet/articulo?codigo $=3824559$

(22) Soares MA, Correa I. Lectura de cuentos infantiles como estrategia de humanización en el cuidado del niño encamado en ambiente hospitalario. Invest Educ Enferm [Internet]. 2011 [acceso 15 de mayo de 2017]; 29 (3): 37080. Disponible en: https://dialnet.unirioja.es/servlet/articulo?codigo $=3840862$

(23) Ceribelli C, Nascimento LC, Pacífico SMR, Garcia de Lima RA. La mediación de la lectura como recurso de comunicación con niños hospitalizados. Rev Latinoam Enfermagem [Internet]. 2009 [acceso 15 de mayo de 2017]; 17(1): 81-7. Disponible en: www.scielo.br/rlae

(24) Wilson DK, Hutson SP, Wyatt TH. Exploring the role of digital storytelling in pediatric oncology patients' perspectives regarding diagnosis. A literature review. SAGE Open [Internet]. 2015 [acceso 27 de mayo de 2017]; 1-10Disponible en: http://www.creativecommons.org/licences/by/3.0/

(25) Hartling L, Scott S, Pandya R, Johnson D, Bishop T, Klassen TP. Storytelling as a communication tool for health consumers: development of an intervention for parents of children with croup. Stories to communicate health information. BMC Pediatrics [Internet]. 2010 [acceso 3 de junio de 2017]; 10 (64): 2-10. Disponible en: http://www.biomedcentral.com/1471-2431/10/64

(26) Orihuela-Pérez I, Cuenca-Rodríguez T, García E, Vélez M. Percepción que el niño/a tiene del " Diario de Paula": Cuento informativo sobre la intervencion quirurgica. Evidentia [Internet]. 2010 [acceso 15 de mayo de 2017]; 7 (29). Disponible en: www.index-f.com/evidentia/n29/ev7163.php

ISSN 1695-6141

๑ COPYRIGHT Servicio de Publicaciones - Universidad de Murcia 